\title{
Directed hepatic differentiation from embryonic stem cells
}

\author{
Xuesong Chen ${ }^{1}$, Fanyi Zeng ${ }^{1,2} \bowtie$ \\ ${ }^{1}$ Laboratory of Developmental Biology, Institute of Medical Science, Shanghai Jiao Tong University School of Medicine, \\ Shanghai 200025, China \\ 2 Shanghai Institute of Medical Genetics, Shanghai Jiao Tong University School of Medicine, Shanghai 200025, China \\ \ Correspondence: fzeng@sjtu.edu.cn \\ Received January 31, 2011 Accepted March 1, 2011
}

\begin{abstract}
The liver is the largest internal organ in mammals, and is important for the maintenance of normal physiological functions of other tissues and organs. Hepatitis, cirrhosis, liver cancer and other chronic liver diseases are serious threats to human health, and these problems are compounded by a scarcity of liver donors for transplantation therapies. Directed differentiation of embryonic stem cells to liver cells is a promising strategy for obtaining hepatocytes that can be used for cell transplantation. In vitro hepatocyte differentiation of embryonic stem cells requires a profound understanding of normal development during embryonic hepatogenesis. Here we provide a simple description of hepatogenesis in vivo and discuss directed differentiation of embryonic stem cells into hepatocytes in vitro.
\end{abstract}

KEYWORDS stem cell differentiation, liver development, signaling pathway

\section{INTRODUCTION}

The liver is the largest internal organ in mammals, and is an indispensable organ involved in many metabolic processes such as detoxification, glycogen and protein metabolism, hematopoiesis and blood storage, immune defense, bile secretion and many other functions. Liver contains a variety of cell types, but the majority are hepatocytes, accounting for $70 \%$ of the liver cell population. Hepatocyte dysfunction can cause a range of liver diseases including hepatitis, cirrhosis and hepatocellular carcinoma. Liver disease is a serious threat to human health, and has a particularly significant impact on Asians. Hepatitis B, an infectious disease caused by the hepatitis B virus, is the most significant hepatocyte pathology in China. At present, there are over 350 million carriers of hepatitis B virus in the world (Vivekanandan and Singh, 2010) and nearly 120 million people carrying hepatitis $B$ virus in China. Hepatocellular carcinoma is the third most common cause of cancer death worldwide. About 700,000 people around the world died of liver cancer in 2008 (Ferlay et al., 2010).

Primary issues include the difficulty of detecting liver disease at an early stage, and poor treatment options. Recently, liver transplantation has become a new means of liver disease treatment, but the scarcity of liver donors and low donor recipient matching rates cause bottlenecks in its application. Researchers have turned their attention to the field of stem cell biology in order to explore new ways to treat liver disease using stem cell differentiation in vitro. Many genes and signaling pathways have been reported to regulate hepatogenesis, and researchers have been able to use in vitro cultures to differentiate embryonic stem cells into hepatocyte-like cells based on these molecular mechanisms, and this may provide an important source of cells for liver transplantation.

\section{LIVER DEVELOPMENT IN MICE}

Liver development in mice is a good model for the counterpart human process. During mouse embryonic development, fertilized eggs undergo the first differentiation and form blastocysts on embryonic day 3.5. At embryonic day 4.0, the blastocyst goes through the second differentiation to form primitive endoderm and primitive ectoderm (Gasperowicz and Natale, 2010). The primitive endoderm is destined to form visceral endoderm (VE) and parietal endoderm (PE), and some liver-specific genes are expressed in the cells of VE 


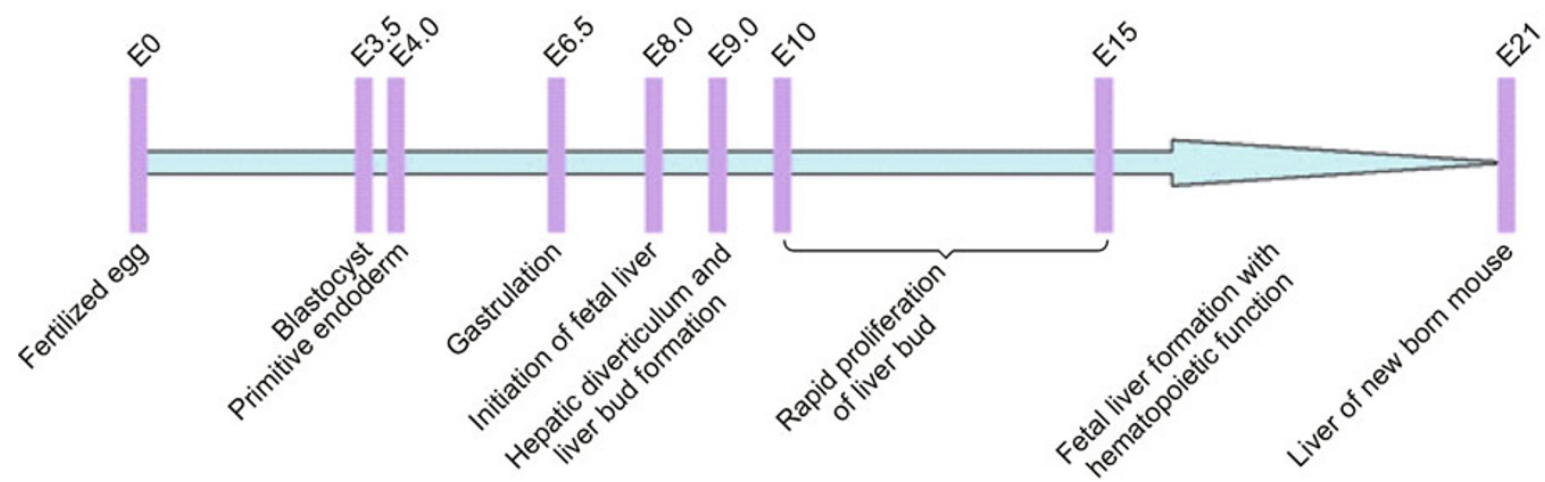

Figure 1. Mouse liver development

such as alpha-fetoprotein (AFP) and transferrin. Visceral endoderm carries out similar functions as liver; however, it never develops into liver or other solid organs. Gastrulation movement initiates at embryonic day 6.5 , and the primitive ectoderm begins to differentiate into endoderm, mesoderm and ectoderm (Beddington and Smith, 1993; Wallingford, 2005). At this stage, the endoderm is termed definitive endoderm. The primitive streak takes shape and consists of foregut, midgut and hindgut regions after gastrulation. The cardiac mesoderm is specified from embryonic day 7.5 (Zaret, 2001). Initiation of the fetal liver is derived from the anterior portion of the definitive endoderm at embryonic day 8.0 (Tremblay and Zaret, 2005). Hepatic diverticula appear, and are the first morphological signal of hepatogenesis. Many signals from cardiac mesoderm and septum transversum mesenchyme (STM), including fibroblast growth factor (FGF) and bone morphogenetic proteins (BMP) signals, are crucial for liver induction (Zaret, 2001; Kung et al., 2010). Hepatoblasts then invade into adjacent STM and have the potential to form both hepatocyte and cholangiocyte. Under the influence of those liver specific induction molecules, hepatoblasts begin to develop into the liver bud from E8.5-9.5. (Douarin, 1975; Houssaint, 1980; Medlock and Haar, 1983) and STM cells also promote the formation of hepatic stellate cells. The liver bud undergoes a rapid proliferation process and becomes the most important hematopoietic organ during embryonic days 10-15 (Fig. 1).

\section{KEY MOLECULES REGULATING HEPATOGENESIS}

As described above, the definitive endoderm at the front of the primitive streak has the capability of developing into liver. At the same time, definitive endoderm cells can also develop into the digestive and respiratory systems. Hepatoblast cells begin to develop towards liver under the influence of induction signals released from the septum transversum mesenchyme and cardiac mesoderm such as FGF and BMP (Zaret, 2001; Kung et al., 2010). In addition to these growth factors, several transcription factors promote specific development to liver
(Zhao et al., 2005). Homeobox protein Hex is expressed in the anterior, in which the common precursors of the liver, gallbladder, pancreas and lung are found (Grapin-Botton, 2005; Moore-Scott et al., 2007). HEX plays a crucial role in liver bud formation and growth. Hex-/- embryos exhibit embryonic lethality because of failed liver formation, and Alb expression is undetectable compared to wild type even in Hex $+/-$ embryos. Kubo et al. have described over-expression of Hex in embryoid body (EB) that induced higher expression of liver specific genes Afp and Alb, demonstrating that Hex has a close relationship with hepatogenesis in vitro (Keng et al., 2000; Kubo et al., 2010). Prox1 is another important molecule taking part in hepatoblast cell migration (Burke and Oliver, 2002; Dudas et al., 2006; Kung et al., 2010).

HNF4 is a nuclear hormone receptor, which belongs to the thyroid/steroid receptor superfamily and influences the expression of many liver specific genes. Hnf4 gene transcription starts from E4.5 in the VE before gastrulation (Chen et al., 1994). HNF4-/- mouse embryos fail to survive to gastrulation as a result of VE dysfunction. A visceral endoderm derived from wild-type tetraploid embryo is able to rescue the embryonic lethal phenotype, indicating that HNF4 is indispensable for early fetal liver development (Watt et al., 2003; Hayhurst et al., 2008). Also, HNF4 can stimulate differentiation of liver cells.

Nodal is a member of the transforming growth factor beta (TGF- $\beta$ ) superfamily, which is important for patterning, mesoderm formation and endoderm regulation and has an especially vital role in gastrulation during embryonic development (Zhou et al., 1993; Conlon et al., 1994). Studies have shown that Nodal gene knockout mice have gastrula defects and failed endoderm development, which eventually lead to developmental arrest at the late gastrula stage. Nodal regulates endoderm and mesoderm development in a concentration dependent manner. Low concentrations can promote the development of mesoderm, and high concentrations are conducive to inner germ layers (endoderm) (Shen, 2007; Zorn and Wells, 2007). At the same time, Nodal mediated signaling pathways have the potential to promote 
expression of the endodermal major transcription factors SOX17 and Foxa1-3 (Zorn and Wells, 2007), and these transcription factors can promote the directional development of the endoderm. The different regions of the primitive streak are distinguished by specific transcription factor expression.

In mammals, the zinc finger transcription factor GATA has isoforms that fall into six families. Molecular genetic analysis showed that GATA factor has the function of controlling the initiation of liver development. In situ hybridization analysis with pluripotent visceral endoderm shows that in the liver bud area, GATA-4 binding to the albumin enhancer occurs upon the initial expression of albumin, indicating that GATA-4 has a role in regulating transcription of liver mRNA (Cirillo et al., 2002). Genetic evidence has indicated that the development of the gastrointestinal tract and its derivatives, including liver, requires regulation of GATA family factors. In the model organism zebrafish, GATA-5 could regulate the developmental process of the intestine and liver (Reiter et al., 1999, 2001); GATA-6 is another candidate factor during liver development, and can regulate the expression of nuclear hormone receptor HNF4 (Morrisey et al., 1998). But, there is no direct evidence of its role in liver development because the absence of GATA6 results in gastrula stage embryo death. Capo-Chichi et al. used an in vitro assay to demonstrate that there are different gene expression levels in GATA-4, GATA-5 and GATA-6 knockout embryonic stem (ES) cells. HNF4 gene expression is high in the GATA-4-/- ES cell line, and AFP expression is high in GATA-5-/- ES cells (Capo-Chichi et al., 2010). By tetraploid compensation analysis in vivo, Zhao et al. have shown that those embryos derived from Gata6-/- embryonic stem cells could only survive to embryonic day 10.5 (Zhao et al., 2005). The results proved that the embryonic lethality of Gata6 specific gene knockout is due to loss of GATA-6 function in the embryo endoderm. The authors analyzed the embryos and found both GATA-4 and GATA- 6 participate in liver specific developmental processes, but GATA-6 is dispensable. However, it is necessary for the rapid proliferation and differentiation of liver bud and mRNA transcription of hepatoblasts which is associated with hepatocyte specificity. The authors also suggest that transiently expressed GATA-4 significantly contributes to hepatic specification and then its expression is lost during the process of hepatic primordium expansion (Zhao et al., 2005).

Kamiya established a new method of fetal liver cell culture in order to study the factors required during the development of liver cells (Kamiya et al., 1999). Their experimental data proved that oncostatin $\mathrm{M}(\mathrm{OSM})$, a cytokine belonging to the IL-6 family and interacting with glucocorticoids, can induce liver cell maturation including the expression of cell markers and the capacity of intracellular glycogen accumulation (Gómez-Lechón, 1999). OSM is expressed in CD45+ hematopoietic cells in the developing liver. Gp130 is an OSM receptor subunit expressed in liver cells, indicating that hepatogenesis is related to a paracrine mechanism and rapid expansion of fetal liver blood cells secreting OSM promotes the development of liver cells in vivo (Miyajima et al., 2000). Kojima et al. elaborated that the livers of gp130 deficient mice have decreased hepatic differentiation marker expression and inefficient glycogenesis (Kojima et al., 2000). Both OSM and interactions between cells are important for liver development and maturation of hepatocytes.

\section{IMPORTANT SIGNALING PATHWAYS INVOLVED IN HEPATOGENESIS}

The key factors driving hepatogenesis are interrelated and play regulatory roles during the development process, creating a signaling network to perform their functions (Fig. 2).

\section{Wnt/ß-catenin}

Both $W n t / \beta$-catenin and TGF- $\beta$ signaling pathways can promote endoderm formation, and the patterning of the endoderm of the primitive streak is influenced by substances secreted from the adjacent mesoderm, such as FGF, Wnt, BMP and retinoic acid (Nejak-Bowen and Monga, 2008). Wnt secreted from mesoderm can inhibit the developmental fate of foregut while promoting the development of the hindgut, so Wnt/ß-catenin and FGF4 signaling pathways must be suppressed during the endoderm development of the anterior foregut (Dessimoz et al., 2006). The foregut area can secrete Sfrp5, which is a Wnt antagonist and can antagonize the Wnt signaling pathway in the foregut region. By this mechanism it promotes the development of mesoderm, which is a key step for inducing the formation of the liver. Therefore, antagonists of Wnt signaling play an indirect role in the induction of liver development. However, at embryonic day 10 the liver bud arises, and $\beta$-catenin (a downstream molecule of Wnt signaling) plays a catalytic role in the rapid growth of liver (Lade and Monga, 2010). Sunny Z. Hussain et al. demonstrated that Wnt-3A played a significant part in liver development by culturing embryonic liver cells in Wnt-3A conditioned culture media.

\section{BMP and FGF signaling}

The signals released from cardiac mesoderm promote proliferation of hepatic endoderm and expression of some liver specific genes. Those septum transversum mesenchyme cells which are next to cardiac mesoderm have the capability of stimulating liver bud outgrowth by secreting certain signals.

The development of mouse liver requires induction both from the FGF signaling of VE and BMP signaling pathway of septum transversum mesenchymal cells and mesoderm (Jung et al., 1999; Rossi et al., 2001). Jung et al. demonstrated that FGFs (1, 2 and 8$)$ derived from cardiac mesoderm 


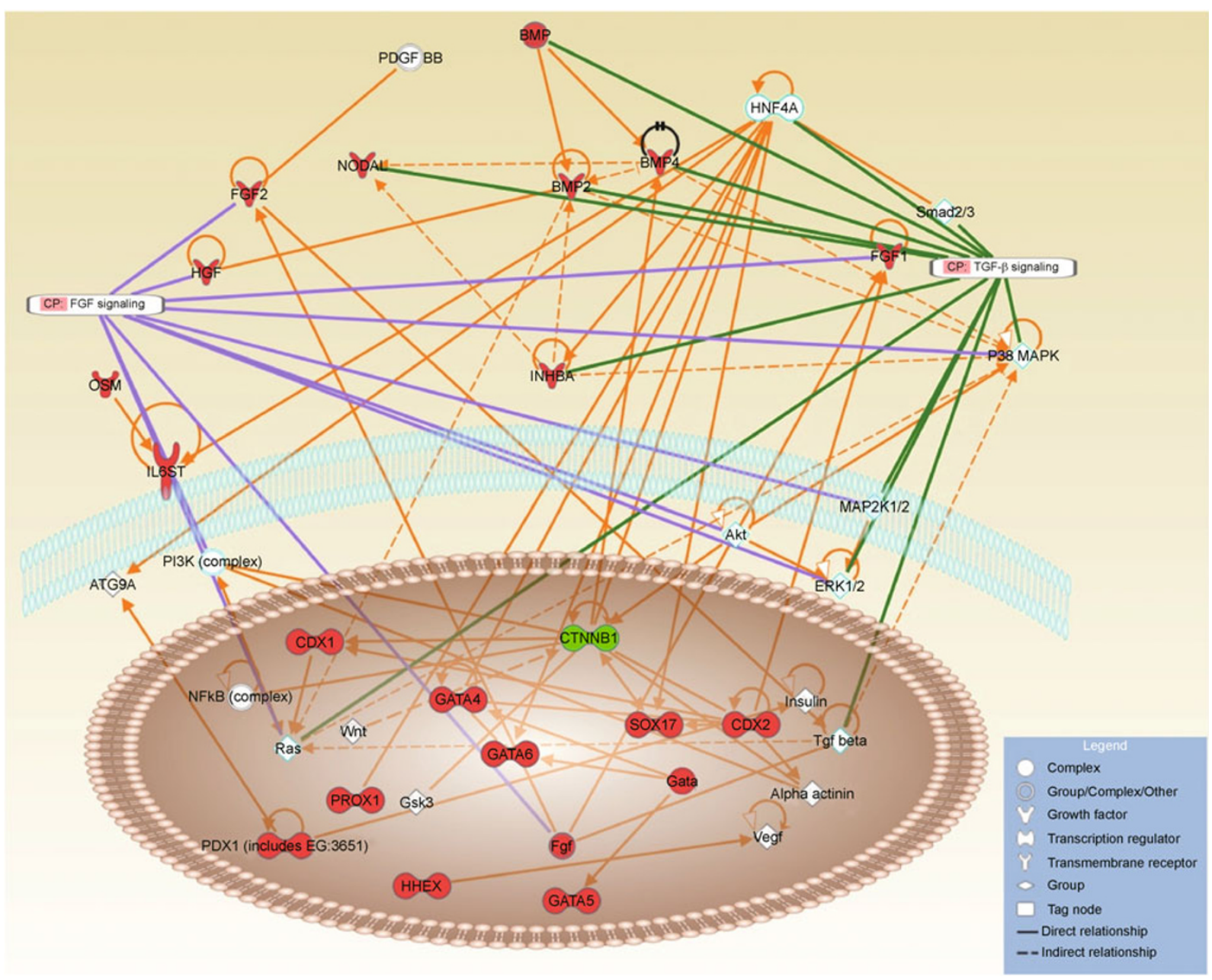

Figure 2. Network of key molecules and signaling pathways related to liver development

are key to liver specific development from forgut endoderm. FGF1 or FGF2 is adequate to induce the isolated mouse foregut endoderm towards hepatogenesis. Meanwhile, FGF8 has the capability of promoting the morphogenenis of hepatic endoderm (Jung et al., 1999; Zaret, 2000). Furthermore, Ameri et al. elaborated that definitive endoderm derived from human embryonic stem cells (ESC) could differentiate into various cell types of forgut lineage under different FGF2 concentrations. A relatively lower concentration of FGF2 favors development into hepatocytes (Ameri et al., 2010). In addition to its role in early liver development, FGF takes part in the regulation of liver bud growth through the phosphatidylinositol 3-kinase (PI3K) signaling pathway.

Many mouse embryonic foregut transplantation experiments have shown that cells cultured from 2-4 somites express albumin after 1-2 days in the presence of the endoderm (Rossi et al., 2001; Calmont et al., 2006). But when the VE is removed or the FGF and BMP signaling pathways are inhibited, the liver induction phenomenon does not occur. Antin and his colleagues also proved that BMP and FGF signaling pathways have the potential of activating hepatocyte-specific gene expression in the chicken embryo (Schultheiss et al., 1997; Zhang et al., 2004). There are some differences between the two species however. BMP2 expressed within the chicken embryo belongs to an autocrine signal, while BMP2 and BMP4 expression occurs in stromal cells in the transverse compartment of the developing mouse, which are paracrine signals.

During the process of liver specification, some key proteins from mesoderm exert a crucial developmental function including BMP2, BMP4 and other BMP protein molecules whose expression is high both in cardiac mesoderm and STM (Rossi et al., 2001; Lemaigre and Zaret, 2004). Bmp4 is expressed in the foregut and hindgut as early as E8.5 and maintains a high expression level in the STM where the liver will migrate. BMP4-/- embryos are embryonic lethal and fail to develop into a distinct liver bud. The liver specific gene albumin can be detected in the tissues of BMP4-/- embryos although they exhibit retarded formation of liver bud (Rossi et al., 2001). 


\section{HGF signaling}

Hepatocyte growth factor (HGF) signaling pathway stimulates hepatoblast cells to proliferate through the tyrosine kinase receptor c-Met, while activation of small GTP enzymes by Arf6 could stimulate hepatoblast migration. HGF is only secreted by mesenchymal cells such as Kupffer cells, not by parenchymal hepatocytes, and takes part in many biological processes including cell growth, mobility and organ morphogenesis (Matsumoto and Nakamura, 1992; Schmidt et al., 1995). Moreover, it plays a mediating role in the development and regeneration of liver by binding with its receptor c-Met. $\mathrm{HGF/c-Met} \mathrm{signaling} \mathrm{is} \mathrm{vital} \mathrm{for} \mathrm{mouse} \mathrm{liver} \mathrm{development,} \mathrm{as}$ confirmed by gene knockout assay. Embryonic mice could not develop completely and died in utero because of lack of HGF expression. In embryonic liver, most parenchymal cells are lost and the overall size of the liver is reduced, combined with impaired placentas (Uehara et al., 1995). HGF is always taken as a hepatotropic factor because expression of HGF increases rapidly in injured livers (Matsumoto and Nakamura, 1992). Paranjpe et al. demonstrated that down regulation of HGF and c-Met by RNA interference in vivo has an influence on proliferation of hepatocytes. Especially, strong c-Met silencing leads to mitosis inhibition and increased apoptosis after partial hepatectomy (Paranjpe et al., 2007). HGF is able to promote DNA synthesis in hepatocytes and increased albumin secretion (Shiota and Kawasaki, 1998).

There are still many other signaling pathways involved in regulating hepatocyte maturation, such as the previously mentioned OSM, which could regulate liver cell maturation through interactions with its receptor gp130 (Gómez-Lechón, 1999; Kamiya et al., 1999; Miyajima et al., 2000; Kamiya et al., 2001).

\section{DIRECTED DIFFERENTIATION OF EMBRYONIC STEM CELLS INTO HEPATOCYTES IN VITRO}

Embryonic stem cells (ESC) are derived from the inner cell mass of blastocysts and have the capability of self-renewal and multilineage differentiation. ESC will differentiate and form an EB after removing feeder cells and culturing in suspension culture medium without leukemia inhibitory factor (LIF) or FGF2; The EBs will spontaneously differentiate into different cell types of the three germ layers including liver cells in adherent culture conditions (Chinzei et al., 2002; Kuai et al., 2003; Asahina et al., 2004; Kumashiro et al., 2005). In order to avoid spontaneous differentiation of ESC forming a mixture of many types of cells, researchers have successfully induced embryonic stem cells to differentiate into liver cells through the reconstruction of an appropriate in vivo microenvironment. The usual methods utilized to direct differentiation into liver cells fall into three categories: (1) soluble factor-induced methods, (2) induction through interaction with different kinds of cells, and (3) induction by chromatin modification. These three methods have their advantages and disadvantages (Table 1), respectively, which are discussed in detail below.

\section{Soluble factor-induced methods}

In 2004, it was reported that mouse embryonic stem cells could be induced in vitro to differentiate into definitive endoderm by adding activin to the culture medium, a method to induce embryoid body formation in suspension culture medium (Kubo et al., 2004). In the following year Baetge et al. induced human embryonic stem cells to differentiate into endoderm cells with a high efficiency of up to $80 \%$ by the same induction method (D'Amour et al., 2005). The researchers found that aFGF and HGF can be used to efficiently induce mouse and monkey embryonic stem cells to differentiate into liver progenitor cells, but they lacked the capabilities of functional mature hepatocytes (Tsukada et al., 2006; Zhou et al., 2007). Then Kume successfully developed an approach of inducing both mouse and human embryonic stem cells into mature hepatocytes by adding activin, bFGF, HGF, OSM, and Dex at different time points based on the original method of inducing embryonic stem cells into pancreatic cells (Agarwal et al., 2008). Lanza optimized Kume's method to differentiate mouse and human ESCs by adjusting the time points and the sequence of induction factors, and the efficiency of producing mature hepatocytes increased to $70 \%-80 \%$ (Shiraki et al., 2008). Basma et al.

Table 1 Comparison of methods to directly induce ESC to form liver cells

$\begin{array}{lll}\begin{array}{l}\text { Induction through } \\ \text { soluble factors }\end{array} & \text { Induction through } & \text { Induction through } \\ & \text { interaction with different } & \text { chromatin modification } \\ \text { kinds of cells } & \end{array}$

kinds of cells

\begin{tabular}{llll}
\hline Advantages & High efficiencies & Mimics the natural & Mimics in vivo liver \\
up to $70 \%-80 \%$ & interactions between \\
hepatocytes & Many heterogeneous cells & $\begin{array}{l}\text { (evelopment, cytokines } \\
\text { are gradually added }\end{array}$ \\
Disadvantages & $\begin{array}{l}\text { increase uncertainties about } \\
\text { the process of directed }\end{array}$ & $\begin{array}{l}\text { are generated during } \\
\text { differentiation }\end{array}$ & $\begin{array}{l}\text { Differentiation } \\
\text { efficiency is unstable } \\
(10 \%-70 \%)\end{array}$
\end{tabular}

ESC, embryonic stem cell. 
were able to obtain functional hepatocytes from human ESCs using a similar protocol (Basma et al., 2009). In 2010, Touboul et al. improved this method with additional factors such as BMP4, FGF10 and retinoic acid, and they produced human hepatocytes exhibiting hepatic functions including glycogen storage, cytochrome activity and low-density lipoprotein uptake (Touboul et al., 2010). There are numerous factors in serum such as hormones and growth factors, which regulate the process of directed differentiation into hepatocytes and yet increase uncertainty of differentiation process at the same time (Jochheim et al., 2004; Saito et al., 2006). Thus many studies focused on serum-free conditions of induction (Agarwal et al., 2008; Hay et al., 2008; Shiraki et al., 2008; Zamule et al., 2011).

\section{Induction through interaction with different kinds of cells}

In addition to the method of adding soluble factors, researchers also found embryonic stem cells could be induced into hepatocytes by co-culture with liver cells or non-liver cells in vitro (Fair et al., 2003; Ishii et al., 2005; Saito et al., 2006). Fetal liver cells were more preferable co-culture cells to promote embryonic stem cell differentiation compared to mesodermal cardiac cells (Fair et al., 2003). Also, the mesenchymal cell line MLSgt20, which is derived from murine fetal livers, was used for directional differentiation of hESCs into hepatocytes in recent work (Ishii et al., 2010). However, the approach of using an artificial microenvironment to mimic the interactions between cells in order to induce liver cell formation would lead to heterogeneous production of many cell types, and therefore high precision instruments are required to separate the target cells from the undesired cells. These methods all aim to reconstruct the microenvironments involved in the process of liver development, including liver induction by signaling molecules released from cardiac mesodermal cells and mesenchymal, parenchymal cells in the liver itself.

\section{Induction by chromatin modification}

The method of changing cell fate by directly modifying chromosomal packaging has advanced in recent years. In 2003, researchers found that liver cells can be enriched from $10 \%-15 \%$ of a population of embryonic stem cells after treatment with sodium butyrate, a histone deacetylase inhibitor (Rambhatla et al., 2003). Cui combined epigenetic modification with cytokines gradually added to the cells in order to induce embryonic stem cells into hepatocytes using sodium butyrate and activin A treatment, achieving a production rate of functional liver cells of $10 \%-70 \%$ (Hay et al., 2008). The induction rate was improved through a novel three-step process with activin A, aFGF and sodium butyrate in recently reported work (Zhou et al., 2010). Sodium butyrate is a histone deacetylase inhibitor which promotes histone acetylation and opening of the chromatin structure; therefore a certain number of vital genes regulating cellular growth and terminal differentiation are activated for transcription (Zhou et al., 2010). Thus chromatin modulation is an important aspect of initiating gene transcription that impacts hepatocyte differentiation.

\section{OUTLOOK}

A number of chronic liver diseases such as hepatitis, cirrhosis and liver cancer can lead to liver failure. There are many issues in liver transplantation treatment for liver failure such as the scarcity of liver donors and recipient immune rejection. Therefore hepatocyte transplantation will be another choice for curing liver failure. There is still a series of problems to be addressed in the process of directed differentiation of embryonic stem cells, including low efficiency and poor quality of available cells. Additionally, other issues affect the use of ESCs (especially human ESCs) in regenerative medicine including immune rejection and ethical problems. Induced pluripotent cells are a better alternative to ES and can be used to derive patient specific hepatocytes for liver transplantation therapy. Developmental biologists are devoted to studying the mechanisms of hepatogenesis and development which will provide a vital foundation for exploring the culture conditions needed for hepatocyte-specific differentiation from embryonic stem cells and induced pluripotent stem (iPS) cells. There will be great clinical value in obtaining mature liver cells with normal physiological functions from embryonic stem cells and iPS cells in vitro.

\section{ACKNOWLEDGEMENTS}

Grant support includes China National Basic Research Program (Grant No. 2007CB947800), the Shanghai Leading Academic Discipline Project (S30201) and STCSM Project (08dj1400502 and 10140900200).

\section{REFERENCES}

Agarwal, S., Holton, K.L., and Lanza, R. (2008). Efficient differentiation of functional hepatocytes from human embryonic stem cells. Stem Cells 26, 1117-1127.

Ameri, J., Ståhlberg, A., Pedersen, J., Johansson, J.K., Johannesson, M.M., Artner, I., and Semb, H. (2010). FGF2 specifies hESCderived definitive endoderm into foregut/midgut cell lineages in a concentration-dependent manner. Stem Cells 28, 45-56.

Asahina, K., Fujimori, H., Shimizu-Saito, K., Kumashiro, Y., Okamura, K., Tanaka, Y., Teramoto, K., Arii, S., and Teraoka, H. (2004). Expression of the liver-specific gene Cyp7a1 reveals hepatic differentiation in embryoid bodies derived from mouse embryonic stem cells. Genes Cells 9, 1297-1308.

Basma, H., Soto-Gutiérrez, A., Yannam, G.R., Liu, L., Ito, R., Yamamoto, T., Ellis, E., Carson, S.D., Sato, S., Chen, Y., et al. (2009). Differentiation and transplantation of human embryonic stem cell-derived hepatocytes. Gastroenterology 136, 990-999. 
Beddington, R.S., and Smith, J.C. (1993). Control of vertebrate gastrulation: inducing signals and responding genes. Curr Opin Genet Dev 3, 655-661.

Burke, Z., and Oliver, G. (2002). Prox1 is an early specific marker for the developing liver and pancreas in the mammalian foregut endoderm. Mech Dev 118, 147-155.

Calmont, A., Wandzioch, E., Tremblay, K.D., Minowada, G., Kaestner, K.H., Martin, G.R., and Zaret, K.S. (2006). An FGF response pathway that mediates hepatic gene induction in embryonic endoderm cells. Dev Cell 11, 339-348.

Capo-Chichi, C.D., Smedberg, J.L., Rula, M., Nicolas, E., Yeung, A.T., Adamo, R.F., Frolov, A., Godwin, A.K., and Xu, X.X. (2010). Alteration of Differentiation Potentials by Modulating GATA Transcription Factors in Murine Embryonic Stem Cells. Stem Cells Int 2010, 602068.

Chen, W.S., Manova, K., Weinstein, D.C., Duncan, S.A., Plump, A.S., Prezioso, V.R., Bachvarova, R.F., and Darnell, J.E. Jr. (1994). Disruption of the HNF-4 gene, expressed in visceral endoderm, leads to cell death in embryonic ectoderm and impaired gastrulation of mouse embryos. Genes Dev 8, 2466-2477.

Chinzei, R., Tanaka, Y., Shimizu-Saito, K., Hara, Y., Kakinuma, S., Watanabe, M., Teramoto, K., Arii, S., Takase, K., Sato, C., et al. (2002). Embryoid-body cells derived from a mouse embryonic stem cell line show differentiation into functional hepatocytes. Hepatology 36, 22-29.

Cirillo, L.A., Lin, F.R., Cuesta, I., Friedman, D., Jarnik, M., and Zaret, K.S. (2002). Opening of compacted chromatin by early developmental transcription factors HNF3 (FoxA) and GATA-4. Mol Cell 9, 279-289.

Conlon, F.L., Lyons, K.M., Takaesu, N., Barth, K.S., Kispert, A., Herrmann, B., and Robertson, E.J. (1994). A primary requirement for nodal in the formation and maintenance of the primitive streak in the mouse. Development 120, 1919-1928.

D'Amour, K.A., Agulnick, A.D., Eliazer, S., Kelly, O.G., Kroon, E., and Baetge, E.E. (2005). Efficient differentiation of human embryonic stem cells to definitive endoderm. Nat Biotechnol 23, 1534-1541.

Dessimoz, J., Opoka, R., Kordich, J.J., Grapin-Botton, A., and Wells, J.M. (2006). FGF signaling is necessary for establishing gut tube domains along the anterior-posterior axis in vivo. Mech Dev 123, 42-55.

Douarin, N.M. (1975). An experimental analysis of liver development. Med Biol 53, 427-455.

Dudas, J., Elmaouhoub, A., Mansuroglu, T., Batusic, D., Tron, K., Saile, B., Papoutsi, M., Pieler, T., Wilting, J., and Ramadori, G. (2006). Prospero-related homeobox 1 (Prox 1 ) is a stable hepatocyte marker during liver development, injury and regeneration, and is absent from "oval cells". Histochem Cell Biol 126, 549-562.

Fair, J.H., Cairns, B.A., Lapaglia, M., Wang, J., Meyer, A.A., Kim, H., Hatada, S., Smithies, O., and Pevny, L. (2003). Induction of hepatic differentiation in embryonic stem cells by co-culture with embryonic cardiac mesoderm. Surgery 134, 189-196.

Ferlay, J., Shin, H.R., Bray, F., Forman, D., Mathers, C., and Parkin, D. M. (2010). Estimates of worldwide burden of cancer in 2008: GLOBOCAN 2008. Int J Cancer 127: 2893-2917.

Gasperowicz, M., and Natale, D.R. (2010). Establishing Three Blastocyst Lineages-Then What? Biol Reprod, Dec 1. [Epub ahead of print]. doi: 10.1095/biolreprod.110.085209

Gómez-Lechón, M.J. (1999). Oncostatin M: signal transduction and biological activity. Life Sci 65, 2019-2030.

Grapin-Botton, A. (2005). Antero-posterior patterning of the vertebrate digestive tract: 40 years after Nicole Le Douarin's PhD thesis. Int J Dev Biol 49, 335-347.

Hay, D.C., Zhao, D., Fletcher, J., Hewitt, Z.A., McLean, D., Urruticoechea-Uriguen, A., Black, J.R., Elcombe, C., Ross, J.A., Wolf, R., et al. (2008). Efficient differentiation of hepatocytes from human embryonic stem cells exhibiting markers recapitulating liver development in vivo. Stem Cells 26, 894-902.

Hayhurst, G.P., Strick-Marchand, H., Mulet, C., Richard, A.F., Morosan, S., Kremsdorf, D., and Weiss, M.C. (2008). Morphogenetic competence of HNF4 alpha-deficient mouse hepatic cells. J Hepatol 49, 384-395.

Houssaint, E. (1980). Differentiation of the mouse hepatic primordium. I. An analysis of tissue interactions in hepatocyte differentiation. Cell Differ 9, 269-279.

Ishii, T., Yasuchika, K., Fujii, H., Hoppo, T., Baba, S., Naito, M., Machimoto, T., Kamo, N., Suemori, H., Nakatsuji, N., et al. (2005). In vitro differentiation and maturation of mouse embryonic stem cells into hepatocytes. Exp Cell Res 309, 68-77.

Ishii, T., Yasuchika, K., Fukumitsu, K., Kawamoto, T., KawamuraSaitoh, M., Amagai, Y., Ikai, I., Uemoto, S., Kawase, E., Suemori, $\mathrm{H}$., et al. (2010). In vitro hepatic maturation of human embryonic stem cells by using a mesenchymal cell line derived from murine fetal livers. Cell Tissue Res 339, 505-512.

Jochheim, A., Hillemann, T., Kania, G., Scharf, J., Attaran, M., Manns, M.P., Wobus, A.M., and Ott, M. (2004). Quantitative gene expression profiling reveals a fetal hepatic phenotype of murine ES-derived hepatocytes. Int J Dev Biol 48, 23-29.

Jung, J., Zheng, M., Goldfarb, M., and Zaret, K.S. (1999). Initiation of mammalian liver development from endoderm by fibroblast growth factors. Science 284, 1998-2003.

Kamiya, A., Kinoshita, T., Ito, Y., Matsui, T., Morikawa, Y., Senba, E., Nakashima, K., Taga, T., Yoshida, K., Kishimoto, T., et al. (1999). Fetal liver development requires a paracrine action of oncostatin $M$ through the gp130 signal transducer. EMBO J 18, 2127-2136.

Kamiya, A., Kinoshita, T., and Miyajima, A. (2001). Oncostatin M and hepatocyte growth factor induce hepatic maturation via distinct signaling pathways. FEBS Lett 492, 90-94.

Keng, V.W., Yagi, H., Ikawa, M., Nagano, T., Myint, Z., Yamada, K., Tanaka, T., Sato, A., Muramatsu, I., Okabe, M., et al. (2000). Homeobox gene Hex is essential for onset of mouse embryonic liver development and differentiation of the monocyte lineage. Biochem Biophys Res Commun 276, 1155-1161.

Kojima, N., Kinoshita, T., Kamiya, A., Nakamura, K., Nakashima, K., Taga, T., and Miyajima, A. (2000). Cell density-dependent regulation of hepatic development by a gp130-independent pathway. Biochem Biophys Res Commun 277, 152-158.

Kuai, X.L., Cong, X.Q., Li, X.L., and Xiao, S.D. (2003). Generation of hepatocytes from cultured mouse embryonic stem cells. Liver Transpl 9, 1094-1099.

Kubo, A., Kim, Y.H., Irion, S., Kasuda, S., Takeuchi, M., Ohashi, K., Iwano, M., Dohi, Y., Saito, Y., Snodgrass, R., et al. (2010). The homeobox gene Hex regulates hepatocyte differentiation from embryonic stem cell-derived endoderm. Hepatology 51, 633-641.

Kubo, A., Shinozaki, K., Shannon, J.M., Kouskoff, V., Kennedy, M., Woo, S., Fehling, H.J., and Keller, G. (2004). Development of 
definitive endoderm from embryonic stem cells in culture. Development 131, 1651-1662.

Kumashiro, Y., Teramoto, K., Shimizu-Saito, K., Asahina, K., Teraoka, H., and Arii, S. (2005). Isolation of hepatocyte-like cells from mouse embryoid body cells. Transplant Proc 37, 299-300.

Kung, J.W., Currie, I.S., Forbes, S.J., and Ross, J.A. (2010). Liver development, regeneration, and carcinogenesis. J Biomed Biotechnol 2010, 984248.

Lade, A.G., and Monga, S.P. (2010). Beta-catenin signaling in hepatic development and progenitors: Which way does the WNT blow? Dev Dyn, Dec 23. [Epub ahead of print]. doi: 10.1002/dvdy.22522

Lemaigre, F., and Zaret, K.S. (2004). Liver development update: new embryo models, cell lineage control, and morphogenesis. Curr Opin Genet Dev 14, 582-590.

Matsumoto, K., and Nakamura, T. (1992). Hepatocyte growth factor: molecular structure, roles in liver regeneration, and other biological functions. Crit Rev Oncog 3, 27-54.

Medlock, E.S., and Haar, J.L. (1983). The liver hemopoietic environment: I. Developing hepatocytes and their role in fetal hemopoiesis. Anat Rec 207, 31-41.

Miyajima, A., Kinoshita, T., Tanaka, M., Kamiya, A., Mukouyama, Y., and Hara, T. (2000). Role of Oncostatin M in hematopoiesis and liver development. Cytokine Growth Factor Rev 11, 177-183.

Moore-Scott, B.A., Opoka, R., Lin, S.C., Kordich, J.J., and Wells, J.M. (2007). Identification of molecular markers that are expressed in discrete anterior-posterior domains of the endoderm from the gastrula stage to mid-gestation. Dev Dyn 236, 1997-2003.

Morrisey, E.E., Tang, Z., Sigrist, K., Lu, M.M., Jiang, F., Ip, H.S., and Parmacek, M.S. (1998). GATA6 regulates HNF4 and is required for differentiation of visceral endoderm in the mouse embryo. Genes Dev 12, 3579-3590.

Nejak-Bowen, K., and Monga, S.P. (2008). Wnt/beta-catenin signaling in hepatic organogenesis. Organogenesis 4, 92-99.

Paranjpe, S., Bowen, W.C., Bell, A.W., Nejak-Bowen, K., Luo, J.H., and Michalopoulos, G.K. (2007). Cell cycle effects resulting from inhibition of hepatocyte growth factor and its receptor c-Met in regenerating rat livers by RNA interference. Hepatology 45 , 1471-1477.

Rambhatla, L., Chiu, C.P., Kundu, P., Peng, Y., and Carpenter, M.K. (2003). Generation of hepatocyte-like cells from human embryonic stem cells. Cell Transplant 12, 1-11.

Reiter, J.F., Alexander, J., Rodaway, A., Yelon, D., Patient, R., Holder, N., and Stainier, D.Y. (1999). Gata5 is required for the development of the heart and endoderm in zebrafish. Genes Dev 13, 2983-2995.

Reiter, J.F., Kikuchi, Y., and Stainier, D.Y. (2001). Multiple roles for Gata5 in zebrafish endoderm formation. Development 128, 125-135.

Rossi, J.M., Dunn, N.R., Hogan, B.L., and Zaret, K.S. (2001). Distinct mesodermal signals, including BMPs from the septum transversum mesenchyme, are required in combination for hepatogenesis from the endoderm. Genes Dev 15, 1998-2009.

Saito, K., Yoshikawa, M., Ouji, Y., Moriya, K., Nishiofuku, M., Ueda, S., Hayashi, N., Ishizaka, S., and Fukui, H. (2006). Promoted differentiation of cynomolgus monkey ES cells into hepatocyte-like cells by co-culture with mouse fetal liver-derived cells. World J Gastroenterol 12, 6818-6827.

Schmidt, C., Bladt, F., Goedecke, S., Brinkmann, V., Zschiesche, W.,
Sharpe, M., Gherardi, E., and Birchmeier, C. (1995). Scatter factor/ hepatocyte growth factor is essential for liver development. Nature 373, 699-702.

Schultheiss, T.M., Burch, J.B., and Lassar, A.B. (1997). A role for bone morphogenetic proteins in the induction of cardiac myogenesis. Genes Dev 11, 451-462.

Shen, M.M. (2007). Nodal signaling: developmental roles and regulation. Development 134, 1023-1034.

Shiota, G., and Kawasaki, H. (1998). Hepatocyte growth factor in transgenic mice. Int J Exp Pathol 79, 267-277.

Shiraki, N., Umeda, K., Sakashita, N., Takeya, M., Kume, K., and Kume, S. (2008). Differentiation of mouse and human embryonic stem cells into hepatic lineages. Genes Cells 13, 731-746.

Touboul, T., Hannan, N.R., Corbineau, S., Martinez, A., Martinet, C., Branchereau, S., Mainot, S., Strick-Marchand, H., Pedersen, R., Di Santo, J., et al. (2010). Generation of functional hepatocytes from human embryonic stem cells under chemically defined conditions that recapitulate liver development. Hepatology 51, 1754-1765.

Tremblay, K.D., and Zaret, K.S. (2005). Distinct populations of endoderm cells converge to generate the embryonic liver bud and ventral foregut tissues. Dev Biol 280, 87-99.

Tsukada, H., Takada, T., Shiomi, H., Torii, R., and Tani, T. (2006). Acidic fibroblast growth factor promotes hepatic differentiation of monkey embryonic stem cells. In Vitro Cell Dev Biol Anim 42, 83-88.

Uehara, Y., Minowa, O., Mori, C., Shiota, K., Kuno, J., Noda, T., and Kitamura, N. (1995). Placental defect and embryonic lethality in mice lacking hepatocyte growth factor/scatter factor. Nature 373 , 702-705.

Vivekanandan, P., and Singh, O.V. (2010). Molecular methods in the diagnosis and management of chronic hepatitis B. Expert Rev Mol Diagn 10, 921-935.

Wallingford, J.B. (2005). Vertebrate gastrulation: polarity genes control the matrix. Curr Biol 15, R414-R416.

Watt, A.J., Garrison, W.D., and Duncan, S.A. (2003). HNF4: a central regulator of hepatocyte differentiation and function. Hepatology 37 , 1249-1253.

Zamule, S.M., Coslo, D.M., Chen, F., and Omiecinski, C.J. (2011). Differentiation of human embryonic stem cells along a hepatic lineage. Chem Biol Interact 190, 62-72.

Zaret, K.S. (2000). Liver specification and early morphogenesis. Mech Dev 92, 83-88.

Zaret, K.S. (2001). Hepatocyte differentiation: from the endoderm and beyond. Curr Opin Genet Dev 11, 568-574.

Zhang, W., Yatskievych, T.A., Baker, R.K., and Antin, P.B. (2004). Regulation of Hex gene expression and initial stages of avian hepatogenesis by Bmp and Fgf signaling. Dev Biol 268, 312-326.

Zhao, R., Watt, A.J., Li, J., Luebke-Wheeler, J., Morrisey, E.E., and Duncan, S.A. (2005). GATA6 is essential for embryonic development of the liver but dispensable for early heart formation. Mol Cell Biol 25, 2622-2631.

Zhou, M., Li, P., Tan, L., Qu, S., Ying, Q.L., and Song, H. (2010). Differentiation of mouse embryonic stem cells into hepatocytes induced by a combination of cytokines and sodium butyrate. J Cell Biochem 109, 606-614.

Zhou, Q.J., Xiang, L.X., Shao, J.Z., Hu, R.Z., Lu, Y.L., Yao, H., and Dai, L.C. (2007). In vitro differentiation of hepatic progenitor cells from mouse embryonic stem cells induced by sodium butyrate. J 
Cell Biochem 100, 29-42.

Zhou, X., Sasaki, H., Lowe, L., Hogan, B.L., and Kuehn, M.R. (1993). Nodal is a novel TGF-beta-like gene expressed in the mouse node during gastrulation. Nature 361, 543-547.

Zorn, A.M., and Wells, J.M. (2007). Molecular basis of vertebrate endoderm development. Int Rev Cytol 259, 49-111. 